Bastian Rauch ${ }^{1}$, Raffaela Calabria ${ }^{2}$, Fabio Chiariello ${ }^{2}$, Patrick Le Clercq ${ }^{1}$, Patrizio Massoli ${ }^{2}$, Michael Rachner ${ }^{1}$

Accurate Analysis of Multicomponent-Fuel Spray Evaporation in Turbulent Flow

Experiments in Fluids Vol. 52 (2012), pp.935-948

${ }^{1}$ Institute of Combustion Technology (DLR), Stuttgart, Germany

${ }^{2}$ Istituto Motori (CNR), Naples, Italy

The original publication is available at www.springerlink.com

http://dx.doi.org/10.1007/s00348-011-1169-0 


\title{
Accurate Analysis of Multicomponent-Fuel Spray Evaporation in Turbulent Flow
}

\author{
Bastian Rauch ${ }^{1}$, Raffaela Calabria ${ }^{2}$, Fabio Chiariello ${ }^{2}$, Patrick Le Clercq ${ }^{1}$, \\ Patrizio Massoli ${ }^{2}$, Michael Rachner ${ }^{1}$ \\ ${ }^{1}$ Institute of Combustion Technology, German Aerospace Center (DLR), Stuttgart, \\ Germany
}

+497116862-210

+497116862-578

bastian.rauch@dlr.de

www.dlr.de/vt/

${ }^{2}$ Istituto Motori, Consiglio Nazionale delle Ricerche (CNR), Naples, Italy

The aim of the paper is to perform an accurate analysis of the evaporation of single component and binary mixture fuels sprays in a hot weakly turbulent pipe flow by means of experimental measurement and numerical simulation. This gives a deeper insight into the relationship between fuel composition and spray evaporation. The turbulence intensity in the test section is equal to $10 \%$, the integral length scale is three orders of magnitude larger than the droplet size while the turbulence microscale (Kolmogorov scales) is of same order as the droplet diameter. The spray produced by means of a calibrated droplet generator was injected in a gas flow electrically preheated. N-nonane, isopropanol and their mixtures were used in the tests. The generalized scattering imaging technique was applied to simultaneously determine size, velocity and spatial location of the droplets carried by the turbulent flow in the quartz tube. The spray evaporation was computed using a Lagrangian particle solver coupled to a gas phase solver. Computations of spray mean diameter and droplet size distributions at different locations along the pipe compare very favorably with the measurement results. This combined research tool enabled further investigation concerning the influencing parameters upon the evaporation process such as the turbulence, droplet internal mixing and liquid phase thermophysical properties.

Spray Evaporation, Multiphase Flow, Spray Diagnostics, Numerical Simulation 


\section{Introduction}

Environmental (IPCC 2007) and security of supply concerns cause an increase in demand for alternative fuels in the transport and energy sectors. This push for a change in fuel production pathways, especially for liquid fuels in the transport sector can yield departures in the fuel composition hence in their thermophysical properties with respect to well known and widely used refined petroleum based fuels. Knowing that models and increasing computing power are available, in design tools, one should shift from single component surrogate with constant properties, to more realistic mixtures. Possibly, this should allow engine designers to reach the optimum in terms of performance and nature-quantity of pollutants under a wide range of fuel oxidation conditions.

Numerical simulation is an important tool for the design of new combustion systems. Such design tools rely on validated sub-models, which themselves rely on accurate experimental studies for their derivation and validation. Furthermore by confronting numerical simulation with experimental data systematic experimental errors can be detected and additional information about the physics of the phenomena are accessible. This defines the overall investigation methodology, which has enabled the accurate analysis of the multiphase-flow under investigation.

When the fuel is supplied in liquid form it has to vaporize prior to burning. Differences in liquid fuel composition can result in differences in local droplet evaporation rate, liquid volume concentrations and vapor mass concentration fields of reacting species and emissions (Le Clercq et al. 2010) in a combustion chamber. Therefore, it is required to take into account the multicomponent nature of the fuels to capture composition's effects on the performance and emission of a combustion system. Specifically, multicomponent-fuel droplet evaporation models allow predicting accurately the times at which volatile components are released in the gas phase. This is of importance when assessing threshold operational situation like high-altitude relight or engine cold start (Caines et al. 2001; Mastorakos 2009).

The evaporation behavior of isolated pure species droplet (suspended) has been extensively investigated (Birouk et al. 2006; Law 1982). Less data is available for 
the similar set-ups but using real fuels. Experimental studies concerning real fuel sprays (monodisperse or polydisperse) with boundary conditions as accurately defined as those for isolated droplet yet with more realistic surrounding conditions, such as hot turbulent air flow, are limited (Ochs et al. 2001; Sommerfeld and Qiu.1998).

The objective of the present paper is to study fuel spray evaporation of multicomponent fuels in simplified yet semi-realistic conditions. Actually, the conditions are simplified with respect to standard aero-engine operating conditions, but with controlled boundary conditions to be able to perform accurate computations. By utilizing experimental methods together with numerical simulation it is aimed at gaining a detailed understanding of the investigated phenomena and its influencing parameters. A generic experiment was built where a monodisperse spray close to ambient temperature is injected into a preheated weakly turbulent flow at ambient pressure. All boundary conditions required for the computations were measured accurately. To characterize the spray evaporation the generalized scattering imaging technique (Calabria et al. 2000) was applied to simultaneously determine size, velocity and, spatial location of the evaporating droplets. This technique has the advantage that it is weakly dependent upon the refractive index; neglecting the refractive index for the droplet sizing yields a measurement uncertainty smaller than $4 \%$ for the droplet sizing. Thus it is possible to characterize fuels with complex composition hence unknown thermophysical properties. The combination of experimental measurement and numerical simulation is used to assess the capability of the combined methods in resolving differences in the spray evaporation due to changes in the composition of the fuels. Moreover, the numerical simulation was used to assess the influence of turbulence, droplet internal mixing and the relative importance of the different thermophysical properties responsible for the penetration length under the present conditions.

\section{Experimental system}

An experimental system was developed to perform accurate measurements of droplet/spray evaporation. The experimental link provides full optical access thereby allowing different kinds of measurement techniques to be employed 
(Rauch et al. 2010). All inflow, outflow, and wall boundary conditions as well as continuous flow and droplet initial conditions required for performing numerical simulations and for validating droplet/spray evaporation models are determined meticulously.

Fig. 1 a) is a schematic representation of the experimental system with the measurement techniques employed. Fig. 1 b) shows in detail the flow conditioning system and the position of the droplet generator. The flow field in the test track is generated by two interacting flows; a preheated weakly turbulent primary flow and a quasi-laminar secondary flow carrying the droplets. Droplets are injected collinearly to the cold secondary gas flow direction. To control inflow conditions especially ensure axisymmetry and generate the turbulence, a combination of honeycombs and perforated plate (hole diameter $1.5 \mathrm{~mm}$, open surface factor $21 \%$ ) are used. In the present configuration, flow velocities of up to $3 \mathrm{~m} / \mathrm{s}$ at a maximum inlet temperature of $800 \mathrm{~K}$ can be reached.

The vibrating orifice aerosol generator (VOAG), Model 3450 of the TSI Corporation, MN, USA, generates a monodisperse stream of droplets. Interchangeable orifices allow varying droplets initial diameter from $21 \mu \mathrm{m}$ to a maximum of about $400 \mu \mathrm{m}$. Then, applying a transversal airflow and forcing the stream through a cone-shaped hole disperses the droplets and generates the monodisperse spray.

(a)

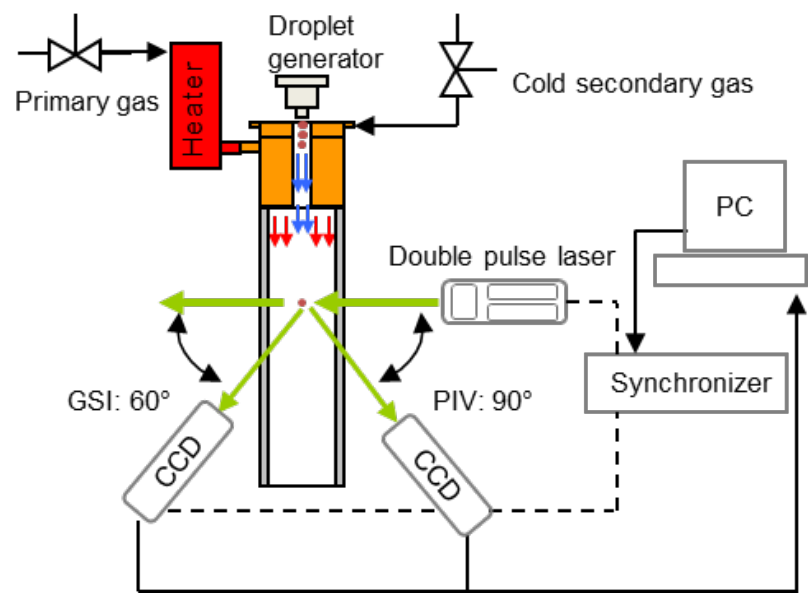




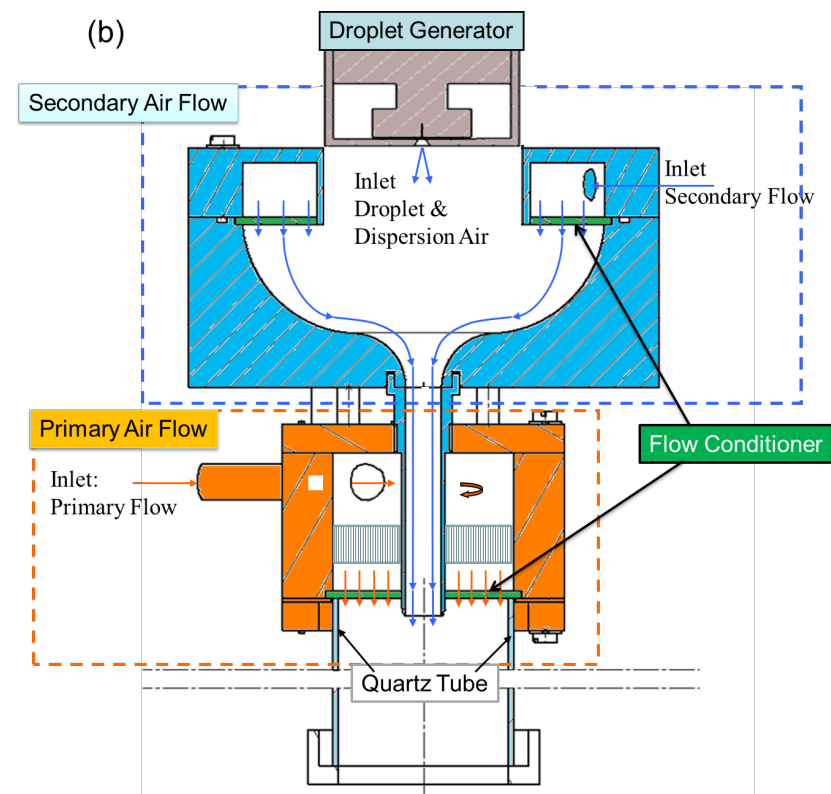

Fig 1 Experimental system with a) applied measurement techniques and b) Inlet system: flow conditioner and droplet generator

Both measurement techniques, the generalized scattering imaging (GSI) for spray characterization and the particle image velocimetry (PIV) for velocity field, were employed in the same experimental set up. In GSI configuration the CCD camera was placed at a scattering angle $\vartheta=60^{\circ}$, where droplets size measurements exhibit the minimum sensitivity to the refractive index. The camera was moved at an angle $\vartheta=90^{\circ}$ for PIV measurements. The generalized scattering imaging technique (Calabria et al. 2000) is used to measure droplet diameter and droplet velocity. GSI is a 2D light scattering sizing technique based on the properties of out-offocus images of droplets captured at a scattering angle of $60^{\circ}$. This technique has the advantage that it is weakly dependent upon the refractive index; neglecting the refractive index the measurement uncertainty is smaller than $4 \%$ for droplet sizing. In case the refractive index of the liquid is known the measurement uncertainty can be reduced to less than $1 \%$. The droplet velocity is inferred from correlating the droplet movement between two laser pulses on two different frames.

The laser is a CFR200 Pulsed Nd:YAG Laser from the company Big Sky Laser Technologies Inc. It has a wavelength of $532 \mathrm{~nm}$, pulse duration of $8 \mathrm{~ns}$ and, repetition rate of 15-30 Hz. The images are taken with the TSI Power View 4MP. The camera has a high resolution with 4 million pixels (resolution $2048 \mathrm{x}$ 
$2048 \mathrm{px}$ ), it has a 12 bit intensity dynamic range and an image capturing rate of 16 frames per second. Variable exposure times and a protective mask are also part of the camera features.

A J-type thermocouple is used for the characterization of the flow temperature field. A fine grid of temperature measurement points was used close to the secondary flow outlet, in the droplet evaporation onset region. In addition, the temperature along the centerline of the secondary flow system was measured, starting from the droplet generator to the secondary flow outlet (i.e.: main flow tube inlet). To complete the set of accurate boundary conditions for the flow field computation, the temperature distribution along the pipe wall and the temperature of the secondary flow system were measured.

\section{Computation}

Spray transport, dispersion and, evaporation processes are computed using the DLR in-house code SPRAYSIM (Le Clercq et al. 2009). It is a simulation tool written in FORTRAN 95 developed at the German Aerospace Center, Institute of Combustion Technology, for spray systems found in premixing/pre-vaporizing modules and gas turbine combustors. SPRAYSIM can generate spray initial conditions based on some state-of-the-art atomization models or fitting of practical sprays initial conditions. Then, it performs the tracking of those generated numerical particles in a Lagrangian framework on unstructured grids and in steady or unsteady gasfields. In the particle's momentum equation the drag force as well as gravity is accounted for. Turbulent particle dispersion is modeled in the present investigation by Blümcke's spectral dispersion model (Blümcke et al. 1993). Simpler dispersion models of Gosman-Ioannides-type are also selectable. The liquid phase can consist of an arbitrary number of discrete and/or continuous (PDF) species. The multicomponent heating and evaporation model is an extension of the single component evaporation model of Abramzon and Sirignano [2]. In the present study single-component droplets of n-nonane and isopropanol as well as their binary (discrete) mixture were simulated. The temperature distribution inside the liquid droplet can be treated in SPRAYSIM either by a uniform temperature model or the conduction limit model or the effective conductivity model of Abramzon and Sirignano (Abramzon et al. 1988). 
Concentration gradients inside the liquid droplet are not accounted for as the rapid mixing model was used here. The system of ordinary differential equations is solved simultaneously for each particle by an Adams-predictor-corrector scheme with automatic internal stepsize control. SPRAYSIM can be used stand-alone or coupled with different gas flow solvers. The data exchange happens by subroutine calls from the gas flow solver or via files. Due to the high dilution rate of the multiphase flow under investigation here SPRAYSIM was used stand-alone. The carrier gas field was computed with the commercial CFD-code ANSYS® CFX12.0. Computational results based on a two-way coupling approach (not shown here) displayed no differences with respect to the one-way coupling computations presented in this paper.

The gas field CFD code solves the Reynolds-Averaged Navier-Stokes (RANS) equations in their conservative form augmented with a transport equation for the specific enthalpy. Additional transport equations for the gaseous species including fuel vapor, for the turbulent kinetic energy and for the turbulent frequency are also solved. Actually, the k- $\omega$ based shear-stress transport (SST) model of Menter (Menter 1994) is used to close the Reynolds stress tensor based on the eddy dissipation concept.

\section{Boundary conditions}

Experiments were performed using a $50 \mu \mathrm{m}$ orifice and a liquid volume flow rate of $0.59 \mathrm{~cm}^{3} / \mathrm{min}$ resulting in a calibrated droplet sprays with a modal value for the diameter between $100 \mu \mathrm{m}$ and $110 \mu \mathrm{m}$. The variation is due to the adjustment of the frequency of the piezoelectric head, which was necessary to guarantee the most stable conditions of operation of the droplet generator. The frequency had to be adjusted for each given liquid and was in the range of 15 to $18 \mathrm{kHz}$. The computational domain is shown in Fig. 2. The flow field boundary conditions are summarized in Table 1 and described in more detail in Rauch et al. 2010. These were kept constant for all measurements. The coordinate system is located at the beginning and on the centerline of the experimental link. This is the first position where measurements could be performed. Droplets and dispersion-air are injected at $[0,0,-131]$ (dimensions are in $\mathrm{mm}$ ) in the direction of the $\mathrm{z}$-axis. 


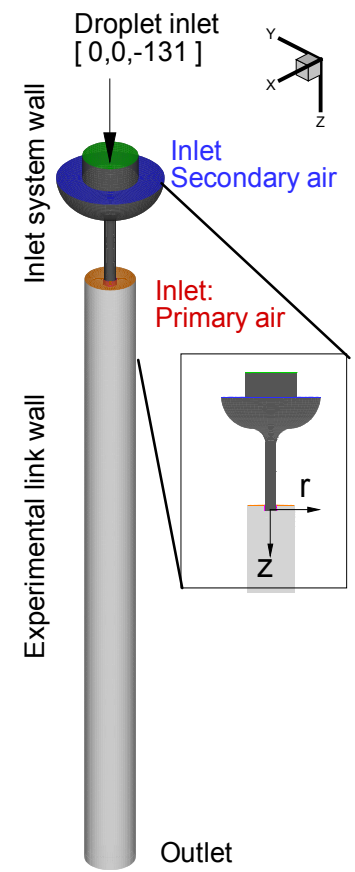

Fig. 2 Computational domain

\begin{tabular}{|l|l|l|}
\hline \multicolumn{2}{|l|}{ Boundary conditions } & Hot flow \\
\hline Inlet system wall & $-131 \mathrm{~mm}<\mathrm{z}<-63 \mathrm{~mm}[\mathrm{~K}]$ & $345 \rightarrow 373$ \\
& $-63 \mathrm{~mm}<\mathrm{z}<-5 \mathrm{~mm}[\mathrm{~K}]$ & 873 \\
& $-5 \mathrm{~mm}<\mathrm{z}<0 \mathrm{~mm}[\mathrm{~K}]$ & 723 \\
\hline Exp. link wall & Constant temperature [K]: & 363 \\
\hline Primary air inlet & Mass flow [kg/s] & $0.983 \times 10^{-3}$ \\
& Temperature & Fig. 3 \\
& Turbulence intensity & $10 \%$ \\
\hline Secondary air inlet & Mass flow [kg/s] & $5.3584 \times 10^{-5}$ \\
& Constant temperature $[\mathrm{K}]$ & 365 \\
& Turbulence intensity & $1 \%$ \\
\hline Droplet dispersion & Mass flow [kg/s] & $2.95 \times 10^{-5}$ \\
air & Constant temperature $[\mathrm{K}]$ & 300 \\
& Turbulence intensity & $1 \%$ \\
\hline
\end{tabular}

Table 1 Flow field boundary conditions

\section{Results}

Tests have been carried out for one set of flow conditions: mean velocity of 1.5 $\mathrm{m} / \mathrm{s}$ and flow inlet temperature of $700 \mathrm{~K}$. N-nonane, isopropanol and their mixtures were used in the tests. The flow field computation results are validated against PIV measurements. Then, spray evaporation computations are performed 
in a pre-computed flow field (one-way coupling) and compared to the spray characteristics measurements.

\section{Flow field validation}

In order to recover a quasi-symmetrical flow field in the test section, a complex flow conditioner was developed, which uses, amongst others, a porous medium layer with a pore size of $25 \mu \mathrm{m}$. Smoke particles from incense sticks were used as seeding material for the PIV measurements. These measurements have been performed by seeding the droplet co-flow (secondary flow) forcing thereby the seeds to pass through the flow conditioner. The smoke particle did not clog the flow conditioner however they could only be used for measurements at room temperature. Thus, the velocity field of the cold flow case was used for the validation of the gas velocity field computations (Rauch et al. 2010) (not shown here). Downstream the incoming laminar secondary (jet) flow ( $\mathrm{z}=[100,250 \mathrm{~mm}])$, the turbulence intensity was measured to be $10 \%$. Hot flow field computations are validated against the measured temperature field and are shown in the following.

(a)

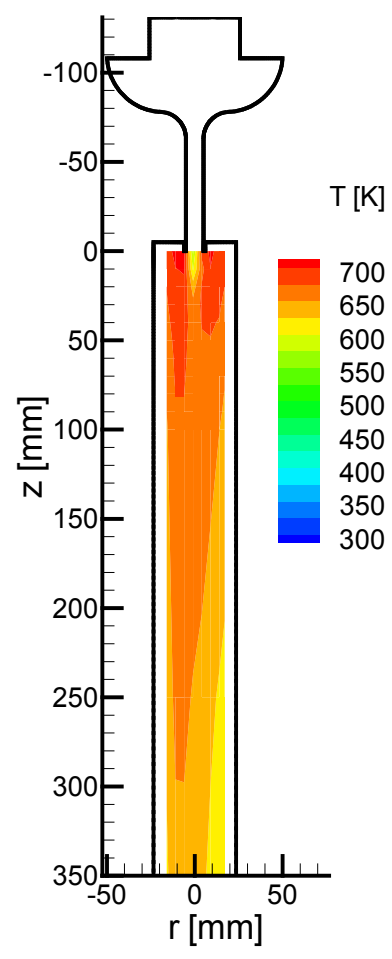

(b)

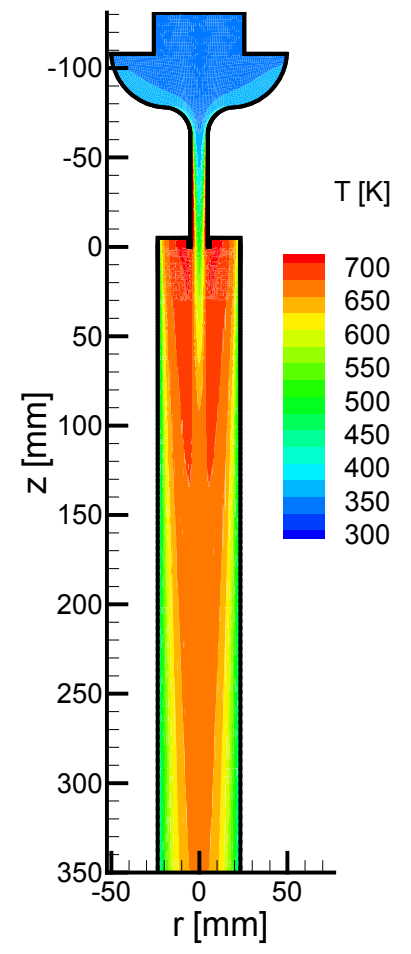

(c)

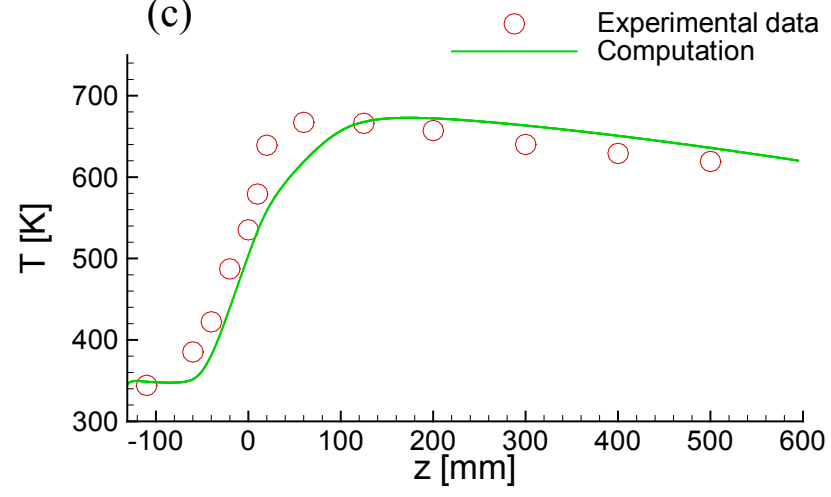


Fig. 3 Hot flow temperature field without spray, (a) experimental data, (b) computation, (c) temperature along the centerline

The computed temperature field displayed in Fig. 3 (b) is in good agreement with the experimental measurement shown in Fig. 3 (a). In Fig. 3 (c), the computed centerline temperature displays a slower temperature increase after $\mathrm{z}=0 \mathrm{~mm}$ with respect to the measurements. This can be explained by the enhanced heat transfer from the surrounding hot primary airflow towards the center due to the transition to turbulence. The computation shows a decline of the temperature rise instead. This is in accordance with the findings from the cold flow simulation (Rauch et al. 2010). In the computations, the transition to turbulence is delayed and less spatially concentrated with respect to the experiment. In addition, a known weakness of turbulence models based on two transport equations (k- $\omega$ for example) is the underestimate of scalar mixing (e.g. temperature field). This explains the weaker heat transfer in the vicinity of axis position $\mathrm{z}=0 \mathrm{~mm}$ observed in the computation (Fig. $3 \mathrm{c}$ ).

\section{Spray analysis: experimental data}

Droplet evaporation measurements have been performed with the pure liquid fuels n-nonane and isopropanol, and their binary mixtures with $30 / 70 \%$ and, $70 / 30 \%$ by volume ratios.

\section{Spray structure}

First, the overall spatial distribution of the spray and its variation along the path are analyzed. To this aim, the size and position of each droplet in the spray at different axial positions were inferred from using the GSI laser diagnostic technique. Fig. 4 shows the reconstruction of the spray spatial distribution for fuels by GSI data in house developed post-processing. Measurements were performed with a $25 \mathrm{~mm}$ step along the $\mathrm{z}$ axis, with the initial location centered at $\mathrm{z}=5 \mathrm{~mm}$, then going down the axis all the way to $\mathrm{z}=230 \mathrm{~mm}$. Each captured droplet is represented by a circle the diameter of which is proportional to its actual size. The height of the spray section in every location is limited by the laser sheet width and is equal to $10 \mathrm{~mm}$. This post-processing method and associated graphical representation allows verifying the centering and the tilting of the spray 
with respect to the quartz tube axis. During the tests, the maximum off-center position and tilting were limited to $2 \mathrm{~mm}$ and $0.5^{\circ}$, respectively.

(a)

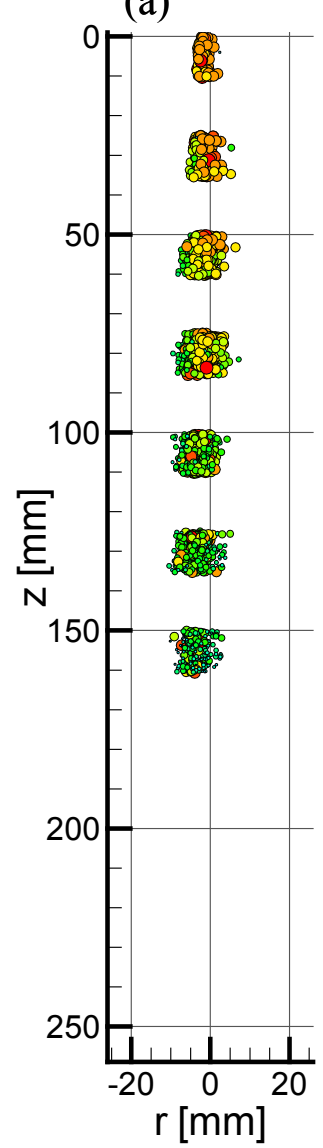

(b)

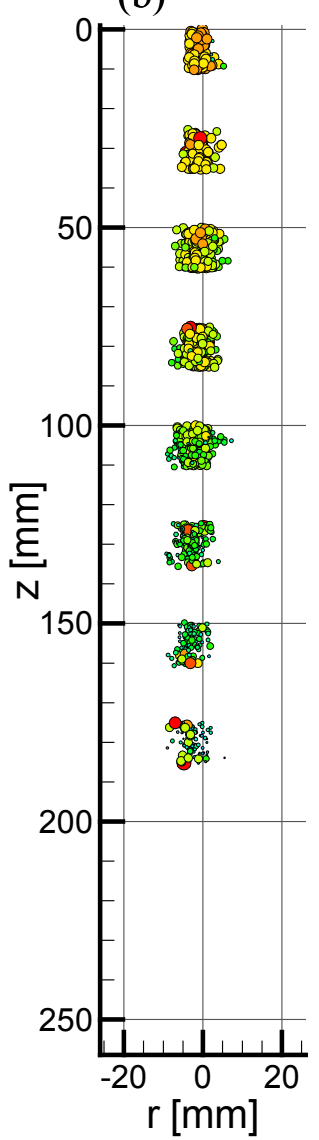

(c)

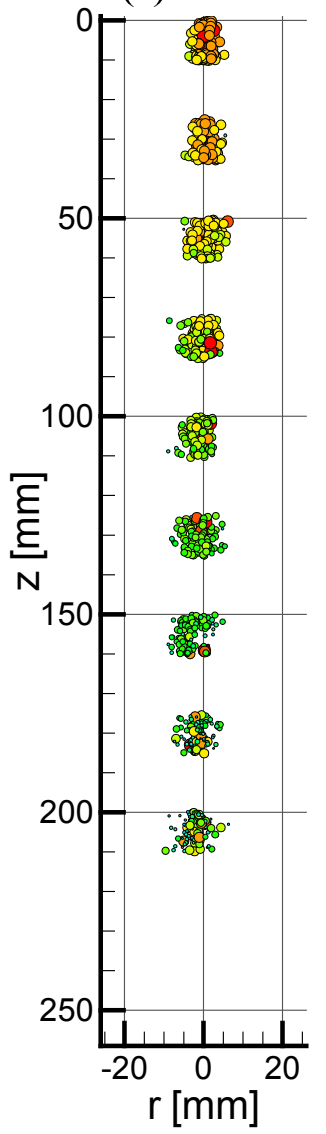

(d)

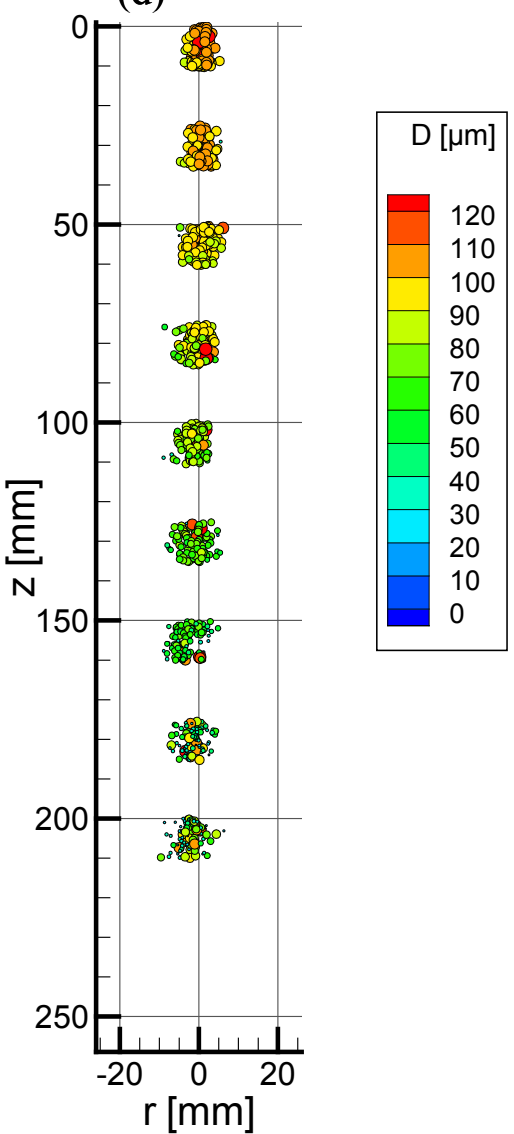

Fig. 4 Droplets size and position distributions after spray reconstruction for (a) n-nonane; (b) 30\% isopropanol / 70\% n-nonane; (c) 70\% isopropanol / 30\% n-nonane; (d) isopropanol. Each individual droplet is represented by a circle with a diameter proportional to the droplet size

From the plots displayed in Fig. 4, one can see that n-nonane is evaporating faster than isopropanol although isopropanol has a lower boiling point and liquid heat capacity with respect to the one of n-nonane. An explanation to this behavior will be given in the last section of this chapter (Influencing parameters) by means of the results of the numerical simulation.

\section{Mean diameter evolution}

From the repeated instantaneous measurements at different axial positions (see Fig. 4), one can derive statistics about the droplet size distribution. On average, 500 droplets have been processed in each measurement position. The spatial evolution of the droplet diameter ensemble average (mean of the distribution) and 
its standard deviation about the mean for the pure fuels and mixtures listed above are given in Fig. 5. Isopropanol droplets (see Fig. 5 d) display the longest evaporation distance. The deviation of droplet diameter distribution from the mean is largest for n-nonane droplets while it is the smallest for isopropanol droplets. The binary component mixtures behave similarly as the pure species, which is in highest concentration.

Mean

Mean (filtered)

std. dev.

std. dev. (filtered)

(a)

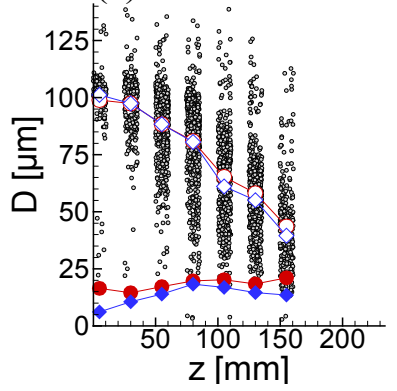

(b)

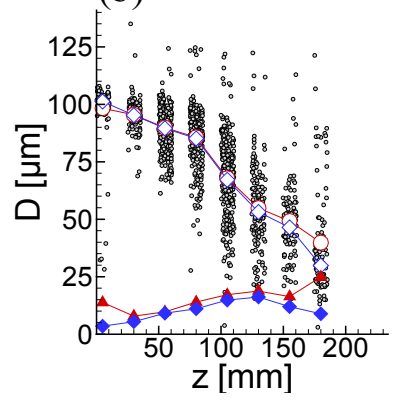

(c)

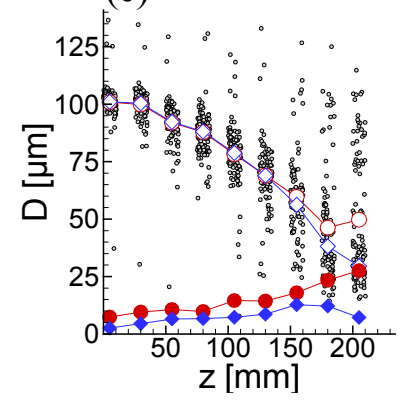

(d)

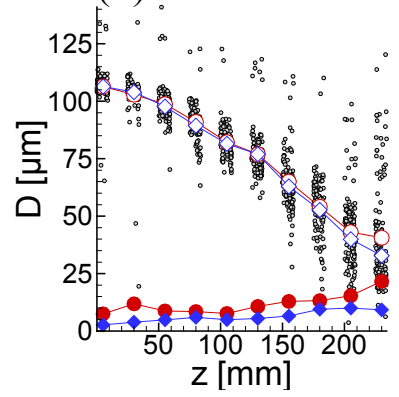

Fig. 5 Droplet diameter dispersion, mean diameter and standard deviation for (a) n-nonane; (b) $30 \%$ isopropanol / 70\% n-nonane; (c) 70\% isopropanol / 30\% n-nonane; (d) isopropanol

Some smaller and bigger droplets outside the normal range of droplet diameters can be observed for all discrete distributions shown in Fig. 5. These can be due to single random effects such as droplet coalescence, to droplet flight paths in the secondary flow that are closer to the near wall hot region, or to droplet generator instabilities (production of satellite droplets), which can result in the production of satellite droplets. For the comparison with the computation these extreme values have been filtered out.

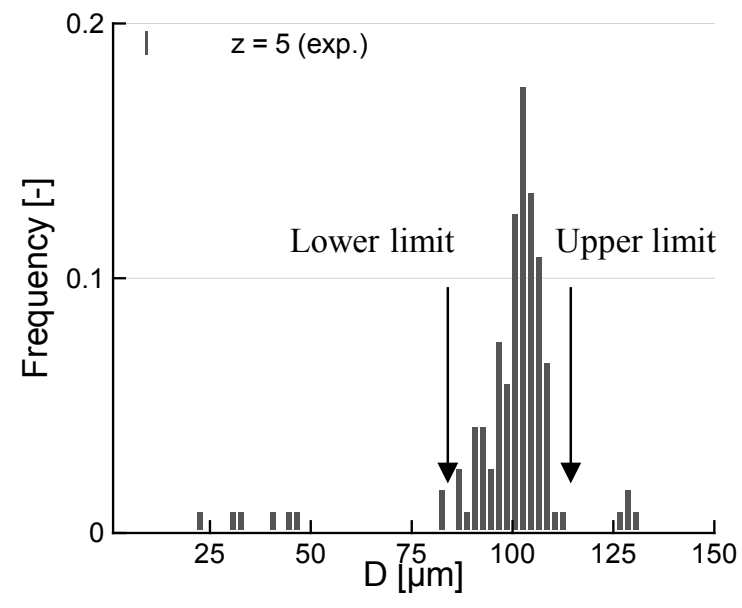


Fig. 6 Diameter distribution and distribution limits identified by the filter at $z=5 \mathrm{~mm}$

Since for the measurements a monodisperse spray is used, the diameter distribution is monomodal. The additional modes which can be seen in Fig. 6 for lower and high diameter ranges are due to random effects. To filter the data the main peak of the distribution function is identified. Starting from this point the two minimum values (threshold value: 0.002 ) in the distribution function, one for the smaller diameters and one for the bigger diameters are identified. Droplets outside this range are removed for the further data analysis.

Moreover, it should be emphasized that the laser sheet width, which is equal to 10 $\mathrm{mm}$ in the axial direction, adds substantially to the size distribution deviation about the mean. Actually, Fig. 7 gives the relevant details concerning the droplet size distributions within the upper and lower halves of the laser sheet separately. As can be seen in Fig. 7 (a), at $\mathrm{z}=80 \mathrm{~mm}$ the n-nonane droplet size distribution in the upper half of the sheet (i.e.: earlier in the droplet lifetime with respect to the middle of the sheet width, see Fig. 7 (b) has a bigger mean value than the distribution in the lower half (i.e.: later in the droplet lifetime). This effect is more pronounced for droplets that vaporize rapidly.

(a)

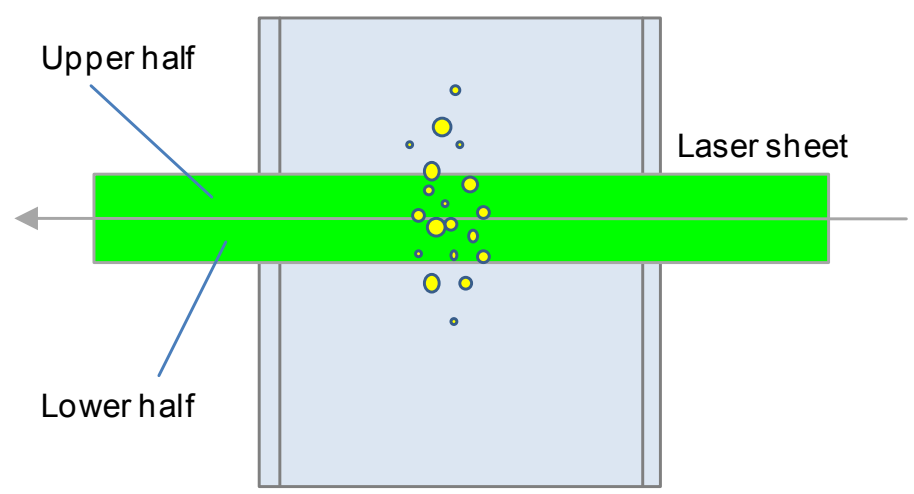

(b)

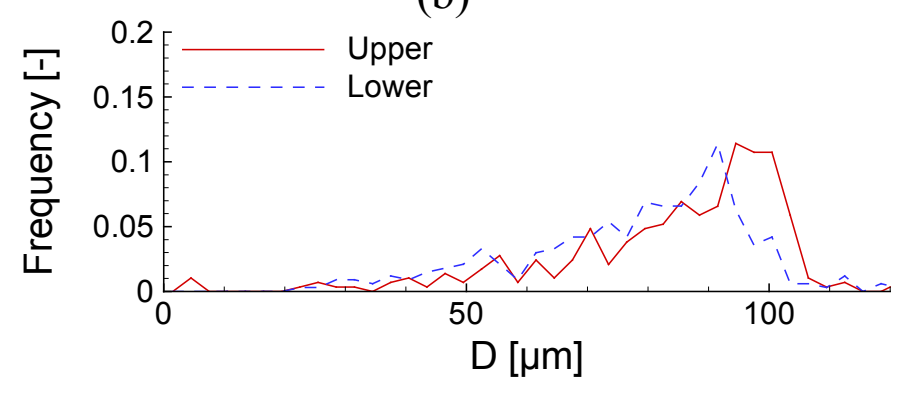


Fig. 7 Effect of averaging over the laser sheet. (a) sketch of the measurement volume; (b) Size distribution of n-nonane droplets at $\mathrm{z}=80 \mathrm{~mm}$ measured in the upper half and lower half regions of the laser sheet

The second effect which has to be analyzed is the uncertainty in the droplet diameter determination due to the laser sheet thickness. In GSI, the size of a droplet is determined by measuring the spacing of the scattering oscillation in outof-focus images (Calabria and Massoli 2000). In the present configuration, the defocus distance was equal $25 \mathrm{~mm}$. However, droplets traversing the beam in different locations will be subject to different magnifications. The laser sheet thickness was $500 \mu \mathrm{m}$ to allow high droplet detection rate. Considering, in first order, a linear effect of the defocusing distance on the image magnification, the expected uncertainty in the size determination due to the laser beam thickness is about $2 \%$. This uncertainty tends to broaden the size distribution toward both extremes.

Finally, the influence of the droplet motion during the image acquisition (exposure time) on the measurement accuracy has been also evaluated. In the present case, the images were frozen by the short duration of the laser pulse. By considering the laser pulse duration, $8 \mathrm{~ns}$, and the maximum droplet velocity, 3 $\mathrm{m} / \mathrm{s}$, the maximum droplet's shift during the image acquisition is equal to $24 \mathrm{~nm}$. Therefore, the maximum deformation of the droplet image is well within the one pixel resolution of the digital system where $1 \mathrm{px}$ corresponds to approximately 10 $\mu \mathrm{m}$. Thus, the finite duration of the laser pulse had negligible influence on the size determination.

\section{Droplet velocities}

In addition to the droplet diameter and droplet position, the GSI technique enables simultaneous measurements of droplet velocities. In Fig. 8, one can see the nnonane droplet velocity field within the laser sheet, which was centered here at the location $\mathrm{z}=5 \mathrm{~mm}$. The main direction of the droplet velocity vector is collinear to the z-axis. 


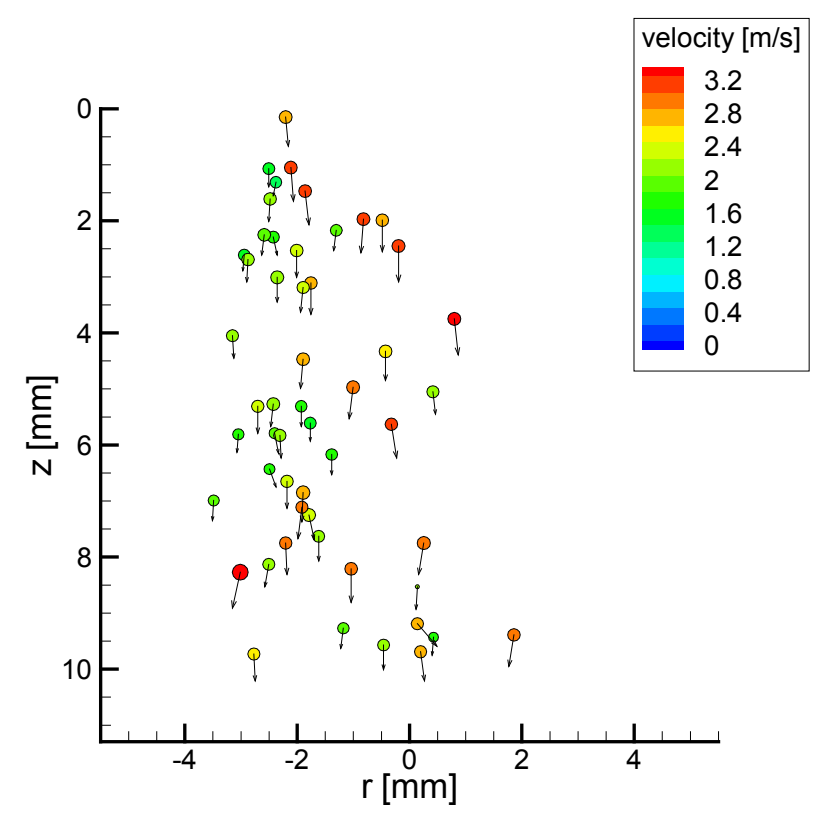

Fig. 8 N-nonane, droplet velocities at $\mathrm{z}=5 \mathrm{~mm}$

For the analysis it is assumed that the flow is axisymmetric to the centerline. The primary flow conditioner was designed to create a homogeneous inflow profile without any radial or tangential velocity components. The honeycombs being part of the system are $13 \mathrm{~mm}$ thick and have a characteristic size of $1 \mathrm{~mm}$, thus any other velocity component except the axial velocity is removed. This is supported by the results of the mean radial droplet velocity shown in Fig. 9. Since the mean value is close to zero, the single droplet radial velocities are due to the turbulent dispersion.

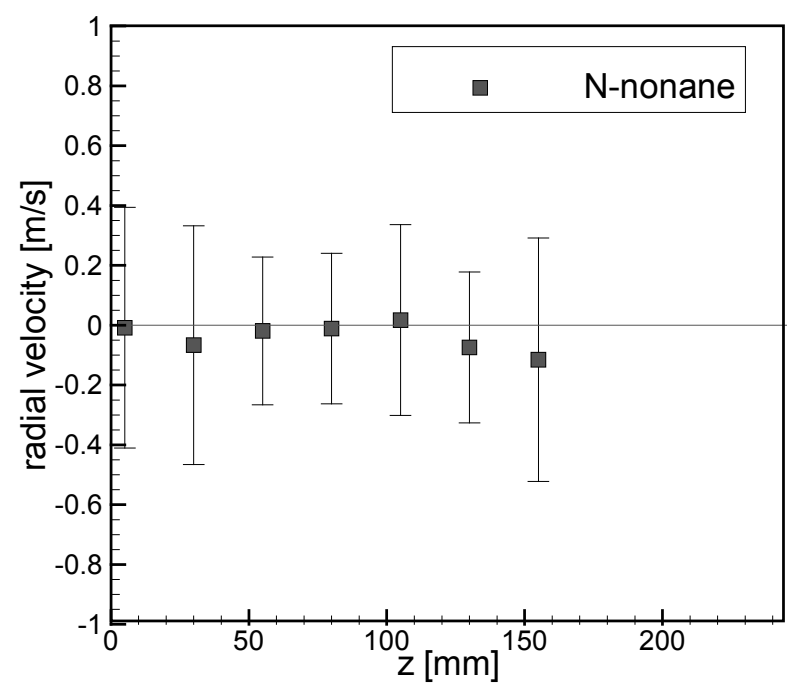

Fig. 9 Plane averaged radial droplet velocity 
Additionally, Fig. 10 displays the droplet diameter as a function of the droplet velocity at different measurement locations. The initial $30 \mathrm{~mm}$ are characterized by a narrow droplet size distribution (around the mean $\mathrm{D}=100 \mu \mathrm{m}$ ) with a relatively wide range of velocities $[1.2-3.2 \mathrm{~m} / \mathrm{s}]$. There, the cloud of points in the plot (Fig. 10, $z=30 \mathrm{~mm}$ ) is mostly vertical. This is due to the larger gradients in the gas flow velocity field (primary-secondary flows mixing layer) with respect to the more uniform downstream region. Downstream, starting at around $\mathrm{z}=55 \mathrm{~mm}$ (see Fig. 10) although the droplet size distribution is substantially wider than in the upstream region $[\mathrm{z}=5-30 \mathrm{~mm}]$, one observes a narrower velocity distribution with respect to the upstream region.
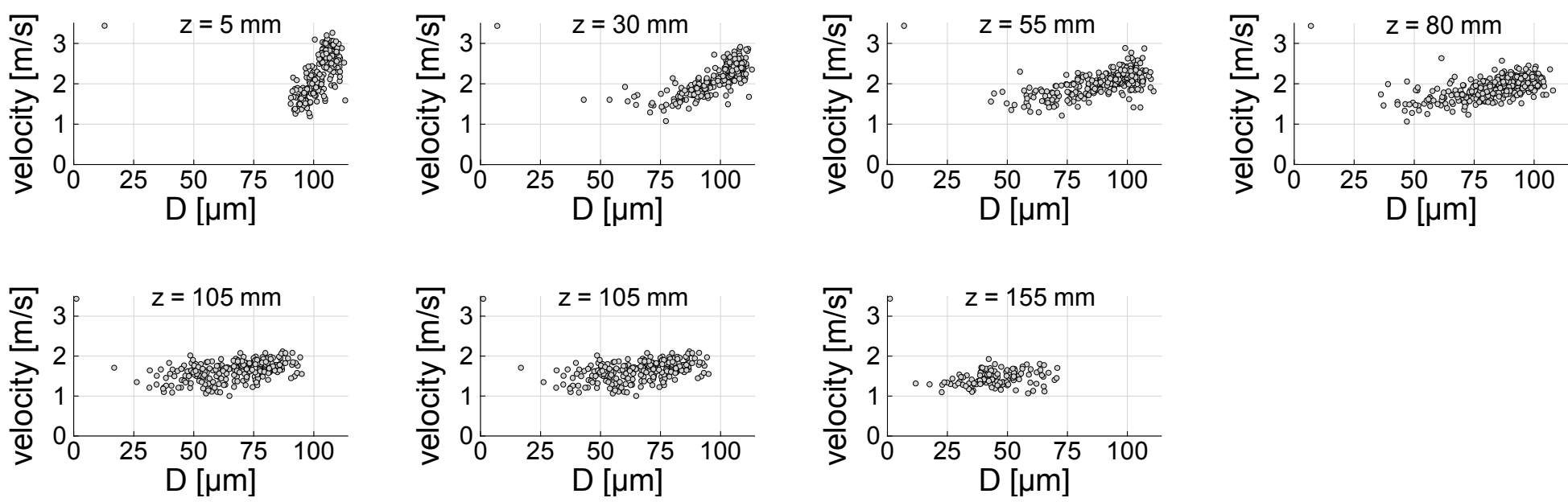

Fig. 10 N-nonane: velocity diameter dependency at the different measurements locations

The Stokes number $\mathrm{St}\left(\mathrm{St}=\tau_{\mathrm{d}} / \tau_{\mathrm{F}}\right)$ is the ratio of droplet relaxation time $\tau_{\mathrm{d}}\left(\tau_{\mathrm{d}}=\rho_{\mathrm{L}} \mathrm{D}^{2} / 18 \mu_{\mathrm{g}}\right)$ and the convective flow characteristic time $\tau_{\mathrm{F}}\left(\tau_{\mathrm{F}}=\mathrm{v} / \mathrm{z}\right)$, where $\mathrm{v}$ is the mean flow field velocity and $\mathrm{z}$ is a characteristic dimension, in our case the travel length of the droplet. The Stokes number was found to be always below 0.05 in test track, consequently the droplets will adapt rapidly to changes in the mean flow. 


\section{Spray analysis: numerical simulation}

\section{Spray initial conditions}

To compute the evaporation of the fuel spray, the experimental size distribution and volume concentration profiles at $\mathrm{z}=5 \mathrm{~mm}$ were used as initial conditions for the SPRAYSIM numerical code. Fig. 11 and Fig. 12 show the initial conditions data extracted from the experiment for n-nonane droplets.

Droplets were injected at the first measurement position, $\mathrm{z}=5 \mathrm{~mm}$, in the experimentally observed off-center position $(-1.9 \mathrm{~mm})$ and with the measured spray radius $(2.1 \mathrm{~mm})$. It was assumed that the spray cloud is spherical symmetric. The droplet temperature and initial composition (in the case of binary mixtures) were estimated by performing computations of the spray heating-up behavior in the secondary flow section before the test track. The droplet initial velocity distribution was computed by taking into account the measured mean velocity and its standard deviation.

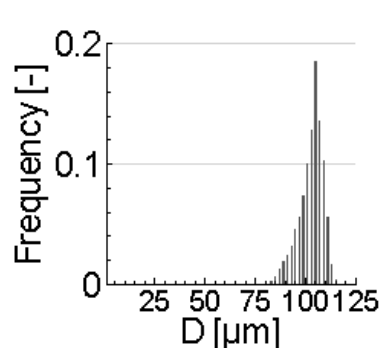

Fig. 11 Initial diameter distribution for $\mathrm{n}$-nonane injection at $\mathrm{z}=5 \mathrm{~mm}$

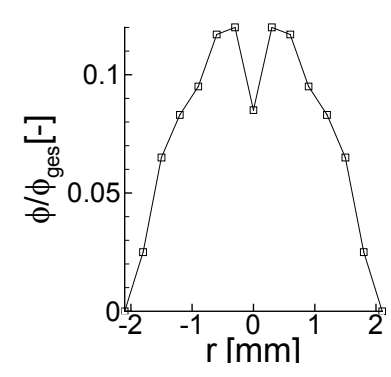

Fig. 12 Volume concentration profile for n-nonane injection at $\mathrm{z}=5 \mathrm{~mm}$

\section{Histogram comparison}

In Fig. 13, the computed histograms for each measurement position are compared with experimental data for n-nonane droplets. The agreement concerning the shape of droplet diameters histograms is very good. In details, one can see that the mean and the width of the computed size distributions agree very well with the measurements. The development of multi-peaked distributions is also qualitatively well captured for axial position below $\mathrm{z}=55 \mathrm{~mm}$. 

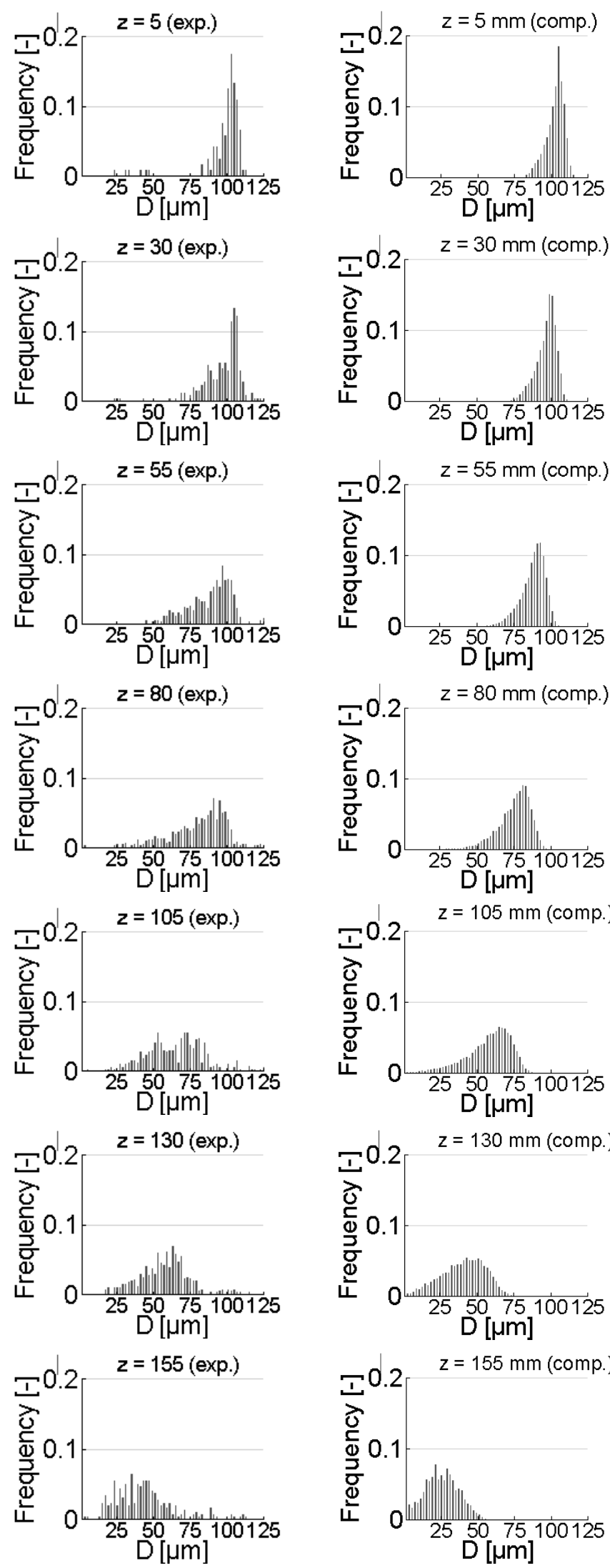

(a)

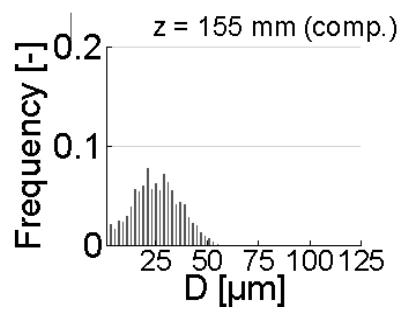

(b)

Fig. $13 \mathrm{~N}$-nonane diameter histogram at each measurement position. (a) experimental data, (b) computations 
In the computations, a slight shift toward smaller diameters occurs between $55 \mathrm{~mm}$ and $80 \mathrm{~mm}$ with respect to the experimental data. This is even more pronounced after $\mathrm{z}=130 \mathrm{~mm}$. The same changes can be seen for the droplet mean velocities (not shown). Further investigations have shown that the droplet co-flow (secondary flow and dispersion air flow see Fig. 1b)) is subject to inlet pressure variations. These fluctuations can have a direct influence on the droplet velocities. The settings used in the actual GSI measurements were not changed during the experimentation. They were tuned to resolve the droplet diameter with high accuracy for most of the droplet lifetime $(20 \mu \mathrm{m}<\mathrm{D}<150 \mu \mathrm{m})$. The lower detection limit of $20 \mu \mathrm{m}$ can be seen in Fig. 13 a) for $\mathrm{z}=130 \mathrm{~mm}$ and $155 \mathrm{~mm}$.

\section{Comparison of mean diameter evolution}

Fig. 14 shows the comparison between the computed and measured mean diameter spatial evolution in the flow direction. Table 2 reports the statistics of the difference from the computed mean diameter to the experimental data. The reported data represent for each fuel the differences averaged over all positions with rejection of the last two. For the statistics the last two measurement positions have been excluded since at these positions the droplets having a diameter below $20 \mu \mathrm{m}$ are not detected by the experimental system, but are representing a considerable fraction of the droplet distribution. Hence the experimental probability distribution function of the diameters measurement error is too affected by the experimental procedure to provide data for a useful comparison. 

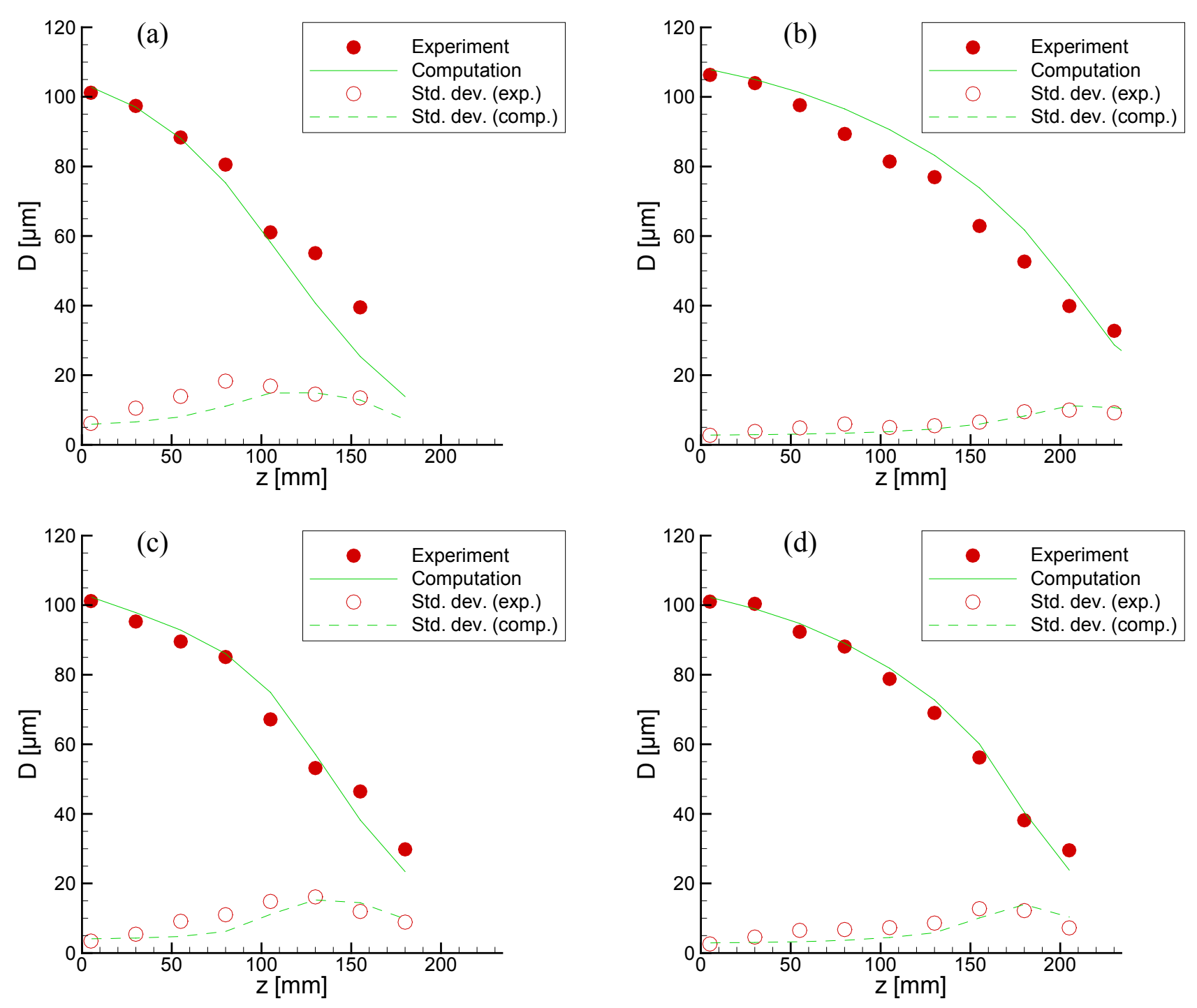

Fig. 14 Comparison of computed and experimental arithmetic mean diameter for (a) n-nonane; (b) isopropanol; (c) 30\% isopropanol / 70\% n-nonane; (d) 70\% isopropanol / 30\% n-nonane

One can see that both the mean diameter and the slope of the curves from the computation results are in very good agreement with the measurements. For nnonane and the binary mixtures the agreement with experimental results is excellent with a difference below $5 \%$. The change in the slope at $\mathrm{z}=80 \mathrm{~mm}$ for the $30 \%$ isopropanol / 70\% n-nonane mixture indicating a volatility-based evaporation process (as opposed to frozen composition) is also very well predicted by the computation. The isopropanol computation shows a good agreement with respect to the experimental data. The predicted droplet diameter toward the end of the penetration length for all fuels is equal to the measured 
value. As mentioned previously, after $\mathrm{z}=55 \mathrm{~mm}$ the measured diameters of $\mathrm{n}$ nonane droplets are shifted toward higher values.

\begin{tabular}{|l|l|l|l|}
\hline Fuel & Mean difference [\%] & Minimum [\%] & Maximum [\%] \\
\hline N-Nonane & 2.6 & 0.3 & 6.5 \\
\hline $\begin{array}{l}70 \% \text { N-Nonane } \\
30 \% \text { Isopropanol }\end{array}$ & 4.6 & 1.1 & 11.5 \\
\hline $\begin{array}{l}30 \% \text { N-Nonane } \\
70 \% \text { Isopropanol }\end{array}$ & 3.2 & 1.1 & 7.0 \\
\hline Isopropanol & 8.2 & 1.0 & 17.4 \\
\hline
\end{tabular}

Table 2 Difference of computed results with respect to experimental data excluding the last two measurement positions

\section{Influencing parameters}

Additional computations have been performed to investigate the influencing parameters on multicomponent-fuel evaporation process and to understand why nnonane droplets evaporate faster than isopropanol droplets (see Fig. 4, Fig. 5 and, Fig. 14). Actually, the effect of turbulence, the influence of internal mixing on the binary mixture fuels evaporation and finally the influence of the thermophysical properties have been studied.

The flow field used for the experiments is characterized by a Reynolds Number of 900. An integral length scale of $1 \sim 10^{-1} \mathrm{~m}$ was estimated by using $1=0.7 \mathrm{r}_{1 / 2}$ (Pope 2000) which is much larger than the droplet size $\left(D=10^{-4} \mathrm{~m}\right)$ and suggests a minor influence on the droplet evaporation (Wu et al 2001). Fig. 15 shows the comparison of the evaporation of n-nonane in a laminar flow field and in the present turbulent flow field. The difference is small and noticeable only past the axis position $\mathrm{z}=70 \mathrm{~mm}$, which corresponds to the shear layer development between primary and secondary flows hence to the highest turbulence intensity region (here 10\%). This difference is comparable to the results of Wu et al 2001. Moreover, since the turbulence integral length scale is three orders of magnitude bigger with respect to the droplet initial diameter it is not the energy-containing turbulent structures which interact with the droplets. Here, droplets are of comparable sizes as the small dissipative structures, which are characterized by 
the Kolmogorov length scale. This translates into a turbulent Stokes number close to unity when adopting the time scale perspective. Thus, for the present flow conditions and liquid properties, we can formulate that weakly turbulent flows have an impact on droplet evaporation when along their trajectory they encounter region of comparable time and length scales as the small turbulence time and length scales.

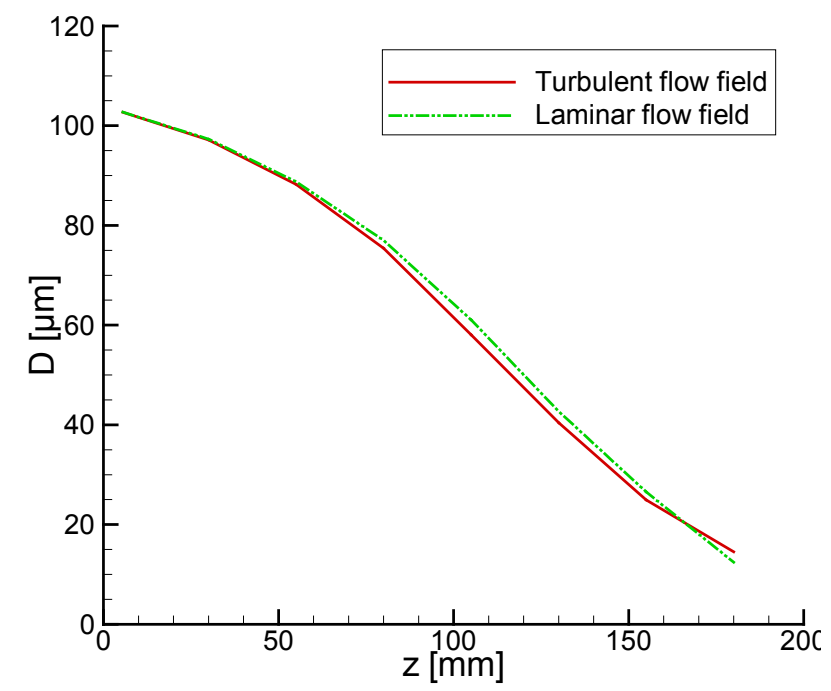

Fig. 15 Comparison of mean diameter evolution for laminar and turbulent flow field conditions.

The model used is in this study is the rapid mixing model where temperature and concentration gradients within the liquid phase are not resolved. To understand the effect of liquid phase mixing, the two limiting cases were used in additional computations, the results of which are presented here after. First, the frozen limit (droplet composition kept constant and equal to its initial composition and heat transport inside the droplet described by the conduction limit model (pure spherically symmetric heat conduction)) and second the rapid mixing (corresponds to infinite mass diffusion and heat conduction). Fig. 16 shows the comparison for the two liquid phase models with the experimental data. The effect of the two different models can be seen only in the later stages of the droplets lifetime. As suggested in Zhang and Law 2008, since these represent the two extreme mixing conditions, the reality lays certainly in between. Note that the present comparison has been carried out for ambient-pressure and moderate temperature conditions. 

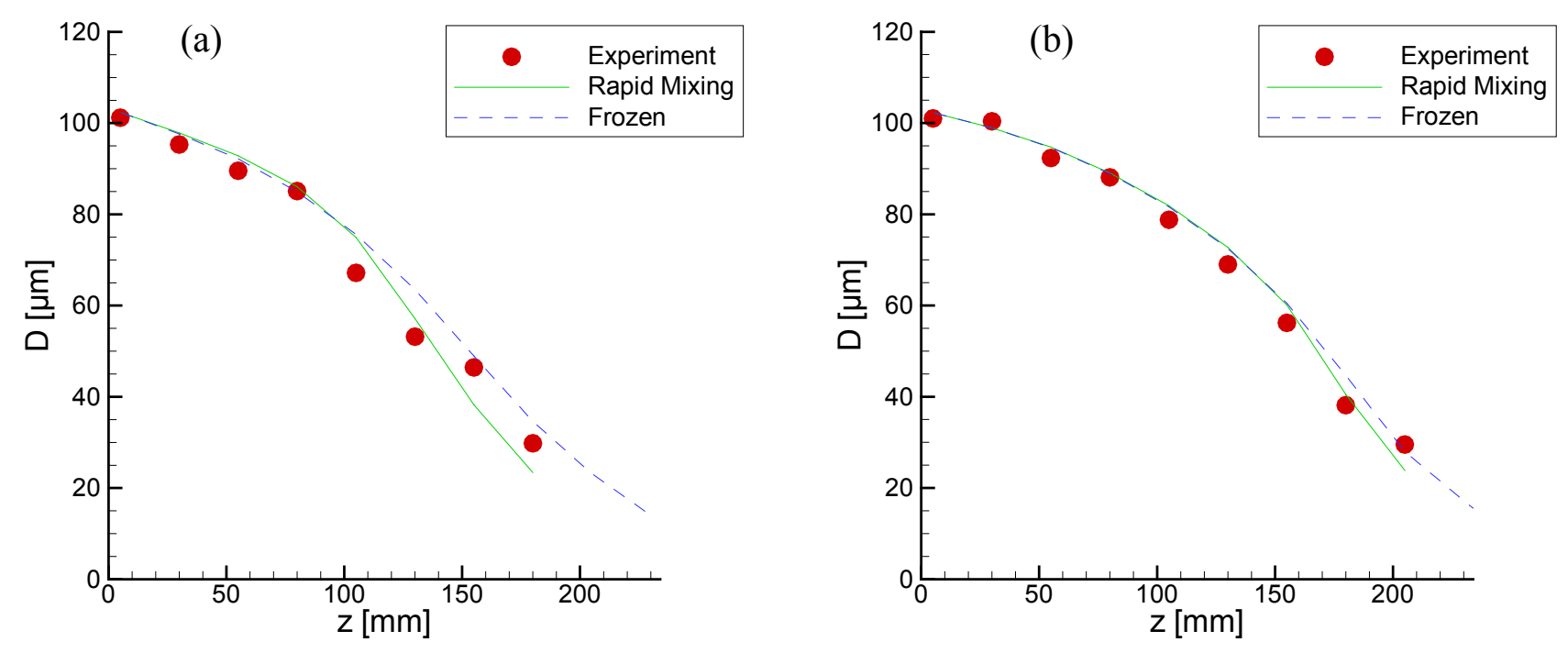

Fig. 16 Comparison of liquid phase models with experimental arithmetic mean diameter for (a) $30 \%$ isopropanol / 70\% n-nonane; (b) 70\% isopropanol / 30\% n-nonane

In order to determine the main physical properties controlling the evaporation in our experiment, simulations with SPRAYSIM have been performed for n-nonane droplets, swapping one thermophysical property at a time to that of isopropanol. The effect of slightly different spray initial conditions at $\mathrm{z}=5 \mathrm{~mm}$ was excluded by setting the initial conditions in the computations of n-nonane equal to those of isopropanol.
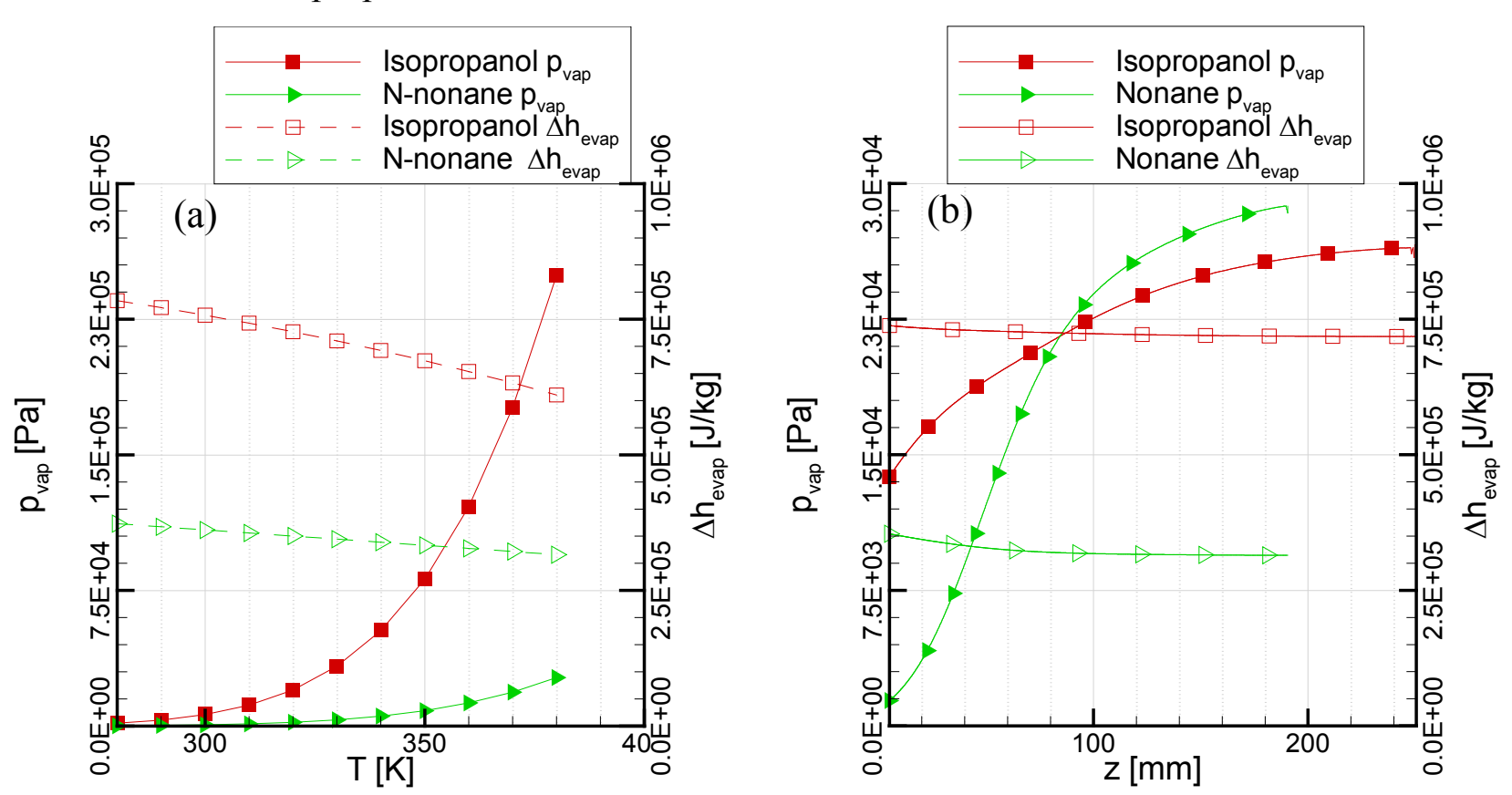

Fig. 17 N-Nonane and Isopropanol: vapor pressure and latent heat of vaporization (a) temperature dependence; (b) evolution along the droplet trajectory 


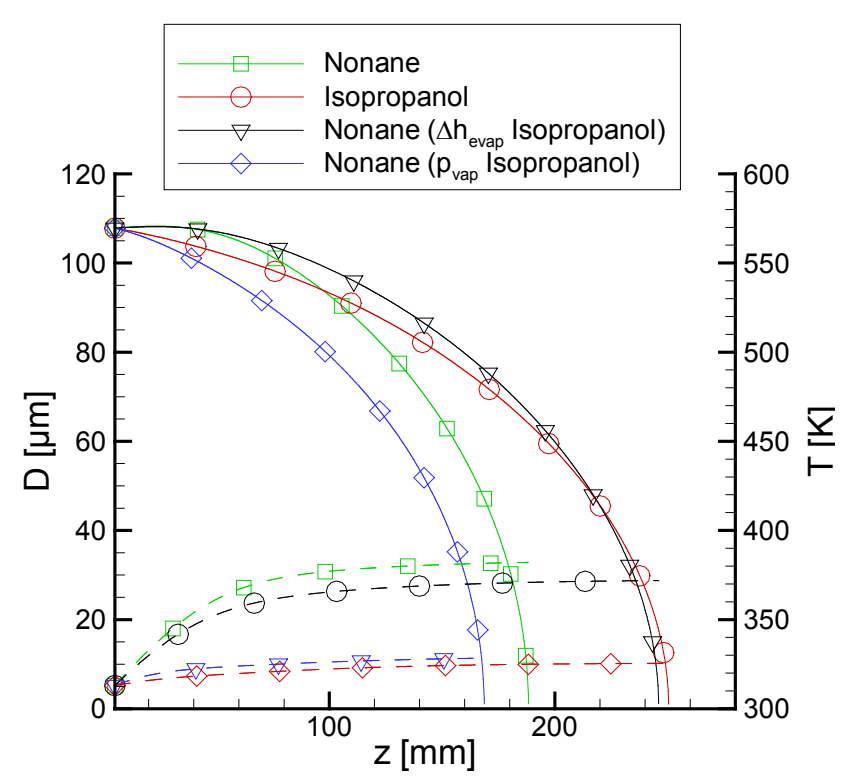

Fig. 18 Diameter and temperature evolution of nonane and adpoted vapor pressure of a single droplet with a trajectory along the centerline

It was found that the heat capacity of the liquids and the binary diffusion coefficient have a negligible influence on the droplet diameter toward the end of the penetration length. Fig. 17 (a) shows a comparison for both fuels of the main physical properties found to be controlling the evaporation, namely the vapor pressure $\mathrm{p}_{\text {vap }}$ and the heat of vaporization $\Delta \mathrm{h}_{\mathrm{evap}}$. The evolution of these properties along the droplet trajectory is shown in Fig. 17 (b). It should be noted that all implemented thermophysical properties are taken from critically evaluated databases (Frenkel et al. 2008; Lemmon et al. 2007) to ensure high data quality. In Fig. 18 the distinct effect of both thermodynamic properties on the diameter evolution and droplet temperature are shown. The vapor pressure at the same temperature is much higher for isopropanol than it is for n-nonane (Fig. 17 a). As expected, the normal boiling point $\mathrm{T}_{\mathrm{nb}}$ is much lower for isopropanol $($ Tnb_isopropanol $=355.4 \mathrm{~K})$ than it is for $n$-nonane $\left(T_{n b \_n o n a n e}=423.9 \mathrm{~K}\right)$. In our case, as the droplet temperature raises to an asymptotic evaporation-end temperature, the so-called wet-bulb temperature, which is always below the boiling temperature at ambient pressure, the n-nonane droplets heat-up to higher temperatures with respect to isopropanol droplets, see Fig. 18. Consequently, the vapor pressure of $\mathrm{n}$-nonane droplets is above that of the colder isopropanol droplets after $\mathrm{z}=85$ mm, see Fig. 17 (b). Therefore, the evaporating mass flow from the n-nonane 
droplets is initially smaller with regard to the one of isopropanol droplets (with same initial temperature), but then becomes larger (steeper dD/dz in Fig. 18) due to the increased droplet temperature. Both droplets approach their wet-bulb temperature at $\mathrm{z} \sim 110 \mathrm{~mm}$ (Fig. 18), where the heating-up phase of the droplet ends. In the beginning of the heating-up phase, the conductive heat flow from the hot ambient gas onto the liquid surface is still large because the counteracting effect of the convective vapor mass flow (Stefan flow) from the surface into the ambient is still small (weak evaporation phase), and because the temperature difference between the hot ambient and the liquid surface (cold droplet) is still large. In that state, the energy transported by the conductive heat flow onto the surface is larger than the energy required for releasing the vapor molecules away from the liquid phase. The heating process in turn leads to an increased evaporating mass flow and lowers the temperature difference between ambient and liquid surface, i.e. the conductive heat flow is diminishing while the energy required for vaporizing the raising vapor mass flow is increasing: constant latent heat $\left(\Delta \mathrm{h}_{\mathrm{evap}}\right)$ times increasing vapor mass flow equals increasing vaporization energy also called heat of evaporation. The result is a continuous decrease of energy remaining for the liquid heating. Finally, if the initial mass of the droplet was sufficiently large, the droplet would have reached the state where this energy sink vanishes. Then, the heating phase would be finished and droplets would have reached their wet-bulb temperature. This state is characterized by a balance between the energy transported by the conductive heat flow onto the surface and the heat of evaporation. This explanation elucidates the important role of the latent heat $[\mathrm{J} / \mathrm{kg}]$ of the liquid in the droplet evaporation process. Indeed, the simulations exhibited that for our case: If $n$-nonane had the same latent heat as isopropanol (which is roughly twice as high as that of n-nonane, Fig. 17), the nnonane droplet would have nearly the same overall penetration length as the isopropanol droplet in our case, as shown in Fig. 18. But as the latent heat of nnonane is actually lower, the penetration length of n-nonane droplets was much smaller than that of isopropanol droplets. In other words: The higher heat of vaporization of isopropanol turned out to be responsible for its slower evaporation compared to n-nonane in our case. 


\section{Conclusions}

Numerical simulations and experimental measurements of evaporating sprays of single component and binary mixtures fuels in a hot turbulent flow were presented. N-nonane, isopropanol and their mixtures were used in the study. The investigation was carried out in an experimental system with controlled fluid dynamics and well defined boundary conditions. The fuel sprays, produced by means of a calibrated droplet generator, were injected in an air flow electrically preheated. The mean flow velocity and inlet temperature used in the experiments were $1.5 \mathrm{~m} / \mathrm{s}$ and $700 \mathrm{~K}$, respectively. Particle image velocimetry was used to determine the gaseous velocity fields. Generalized scattering imaging technique was applied to simultaneously determine size, velocity and spatial location of the evaporating droplets.

The spray transport and evaporation processes were computed with the multiphase-flow simulation tool: SPRAYSIM coupled to ANSYS ${ }^{\circledR}$ CFX-12.0. The computed gas field was validated against measurement data. Computed hot gas flow field were then used for the computation of the spray evaporation. Initial conditions for the spray computation were taken from experimental measurements at $\mathrm{z}=5 \mathrm{~mm}$. The droplet temperature and initial composition was estimated by performing computations of the spray heating-up behavior in the section before the test track. Computed mean diameter and histograms of n-nonane, isopropanol and their mixtures are found to be in very good agreement with the experimental data.

With the validated numerical simulations it was then possible to investigate the effect of turbulence, of internal mixing in the droplet and of the relative importance of the different thermophysical properties under the given experimental conditions. The flow was found to be weakly turbulent and the expected small influence of the turbulence on the evaporation process was found in the highest turbulence intensity $(10 \%)$ region of the present flow. The effect of different droplet mixing models (frozen composition or rapid mixing model) was limited and could only be seen in the later stages of the droplet lifetime. Finally it was shown that the latent heat of vaporization is the controlling factor responsible for the faster evaporation of n-nonane droplets with respect to isopropanol droplets.

The original publication is available at www.springerlink.com 


\section{Acknowledgments}

The research was partially supported by the Italian Ministry of Economic Development within the framework of the Program Agreement MiSE-CNR "Ricerca di Sistema Elettrico". The Juwi funding program of the German Aerospace Center is also greatly acknowledged.

The Applied Physics group at Istituto Motori - CNR in Naples wishes to thank TSI Inc. Minnesota USA for having kindly provided the high resolution PowerView Plus CCD and the Insight $3 G \AA$ software package.

\section{References}

Abramzon B, Sirignano WA (1988) Droplet vaporization model for spray combustion calculations, AIAA-88-0636

Birouk M, and Gökalp I (2006) Current status of droplet evaporation in turbulent flows. Progress in Energy and Combustion Science 32: 408 - 423

Blümcke E, Brandt M, Eickhoff H, Hassa C (1993) Particle dispersion in highly swirling, turbulent flows, Particle \& Particle Systems Characterization 10 : 182-190

Calabria R, and Massoli P (2000) Experimental study of droplets in evaporating regime by 2D scattering analysis. 10th Int. Sympos. on Applications of Laser Techniques to Fluid Mechanics Lisbon, Portugal, 10-13 July

Caines BN, Hicks RA and Wilson CW (2001) Influence of Sub-Atmospheric Conditions on the Performance of an Airblast Atomiser, 37th AIAA/ASME/SAE/ASEE Joint Propulsion Conference, Salt Lake City, UT,8-11 July

Frenkel M, Chirico RD, Diky V, Muzny C, Lemmon EW, Yan X, Dong Q (2008) NIST Standard Reference Database 103a, NIST ThermoData Engine, Version 2.1; Standard Reference Data; National Institute of Standards and Technology: Gaithersburg, MD IPCC (2007) Intergovernmental Panel on Climate Change (IPCC), Fourth Assessment Report. Law CK. (1982) Recent advances in droplet vaporization and combustion. Progress in Energy and Combustion Science 8: 171 - 201

Le Clercq P, Doué N, Rachner M, Aigner M (2009) Validation of a multicomponent-fuel model for spray computations. Proc. 47th AIAA Aerospace Sciences Meeting Orlando, Florida, AIAA2009-1188

Le Clercq P, Di Domenico M, Rachner M, Ivanova E, Aigner M (2010) Impact of FischerTropsch Fuels on Aero-Engine Combustion Performance. Proc. 48th AIAA Aerospace Sciences Meeting Orlando, Florida, AIAA-2010-613

Lemmon EW, Huber ML, McLinden MO (2007) NIST Standard Reference Database 23, NIST Reference Fluid Thermodynamic and Transport Properties Database (REFPROP), Version 8.0; Standard Reference Data; National Institute of Standards and Technology: Gaithersburg, MD, 2007 
Mastorakos E (2009) Ignition of turbulent non-premixed flames, Progress in Energy and Combustion Science 35 No.1: 57 - 97

Menter FR (1994) Two-equation eddy-viscosity turbulence models for engineering applications. AIAA journal 32: 1598 - 1605

Ochs M, Gass J, Reh L (2001) Convective vaporization of freely moving fuel droplets in a hot air flow. ILASS-Europe 2001 Zürich, Switzerland, 2-6 Sept.

Pope S (2000) Turbulent Flows, Cambridge Univ. Press

Rauch B, Chiariello F, Calabria R, Le Clercq P, Massoli P (2010) Prediction of refractive index profiles within evaporating droplets. 12th Workshop on Two-Phase Flow Predictions Halle (Saale), Germany, 22-25 March

Sommerfeld M, Qiu H (1998) Experimental studies of spray evaporation in turbulent flow. International Journal of Heat and Fluid Flow 19: 10 - 22

Wu JS, Liu YJ, Sheen HJ (2001) Effect of ambient turbulence and fuel properties on the evaporation rate of single droplets. Int. J. Heat and Mass Transfer 44:4593-4603

Zhang H, Law CK (2008) Effects of temporally varying liquid-phase mass diffusivity in multicomponent droplet gasification. Combustion and Flame 153: 593 - 602 